\title{
Some results of $\varpi$-Painlevé difference equation
}

\author{
Yong Liu ${ }^{1 *}$ (D) and Yuqing Zhang ${ }^{2}$
}

\section{"Correspondence: liuyongsdu1982@163.com 'Department of Mathematics, Shaoxing College of Arts and Sciences, Shaoxing, China Full list of author information is available at the end of the article}

\section{Abstract}

In this article, we mainly investigate some properties of two types of difference equations

$$
Y(\varpi z)+Y(z)+Y\left(\frac{z}{\varpi}\right)=\frac{\xi z+0}{Y(z)}+v
$$

and

$$
Y(\varpi z)+Y\left(\frac{z}{\varpi}\right)=\frac{\xi z+o}{Y(z)}+\frac{V}{Y^{2}(z)}
$$

MSC: $30 \mathrm{D} 35 ; 39 \mathrm{~B} 12$

Keywords: Rational functions; Complex difference equations; Value distribution; Finite order

\section{Introduction}

Halburd and Korhonen [4] used Nevanlinna theory to single out difference equations in this form

$$
Y(z+1)+Y(z-1)=R(z, Y)
$$

where $R(z, Y)$ is rational in $O$ and meromorphic in $z$, has an admissible meromorphic solution of finite order, then either $O$ satisfies a difference Riccati equation

$$
Y(z+1)=\frac{p(z+1) Y(z)+q(z)}{Y(z)+p(z)}
$$

where $p(z), q(z) \in S(Y)$, where $S(Y)$ denotes the field of small functions with respect to $Y$, or Eq. (1.1) can be transformed to one of the following equations:

$$
\begin{aligned}
& Y(z+1)+Y(z)+Y(z-1)=\frac{\varsigma_{1} z+\varsigma_{2}}{Y(z)}+\kappa_{1}, \\
& Y(z+1)-Y(z)+Y(z-1)=\frac{\varsigma_{1} z+\varsigma_{2}}{Y(z)}+(-1)^{z} \kappa_{1},
\end{aligned}
$$

(c) The Author(s) 2018. This article is distributed under the terms of the Creative Commons Attribution 4.0 International License (http://creativecommons.org/licenses/by/4.0/), which permits unrestricted use, distribution, and reproduction in any medium, provided you give appropriate credit to the original author(s) and the source, provide a link to the Creative Commons license, and indicate if changes were made. 


$$
\begin{aligned}
& Y(z+1)+Y(z-1)=\frac{\varsigma_{1} z+\varsigma_{3}}{Y(z)}+\varsigma_{2} \\
& Y(z+1)+Y(z-1)=\frac{\varsigma_{1} z+\kappa_{1}}{Y(z)}+\frac{\varsigma_{2}}{Y^{2}(z)} \\
& Y(z+1)+Y(z-1)=\frac{\left(\varsigma_{1} z+\kappa_{1}\right) Y(z)+\varsigma_{2}}{(-1)^{-z}-Y^{2}(z)} \\
& Y(z+1)+Y(z-1)=\frac{\left(\varsigma_{1} z+\kappa_{1}\right) Y(z)+\varsigma_{2}}{1-Y^{2}(z)} \\
& Y(z+1) Y(z)+Y(z) Y(z-1)=p \\
& Y(z+1)+Y(z-1)=p Y(z)+q
\end{aligned}
$$

where $\varsigma_{k}, \kappa_{k} \in S(Y)$ are arbitrary finite order periodic functions with period $k$.

Eqs. (1.3), (1.5), and (1.6) are known alternative forms of difference Painlevé I equation, Eq. (1.8) is a difference Painlevé II, and (1.9) and (1.10) are linear difference equations. Chen and Shon [2,3] considered some value distribution problems of finite order meromorphic solutions of Eqs. (1.2), (1.5), (1.6), and (1.8). A natural question is: What is the result if we give q-difference analogues of (1.3) and (1.6)? For this question, we consider the following equations:

$$
\begin{aligned}
& Y(\varpi z)+Y(z)+Y\left(\frac{z}{\varpi}\right)=\frac{\xi z+o}{Y(z)}+v, \\
& Y(\varpi z)+Y\left(\frac{z}{\varpi}\right)=\frac{\xi z+o}{Y(z)}+\frac{v}{Y^{2}(z)} .
\end{aligned}
$$

Theorem 1.1 Let $Y(z)$ be a transcendental meromorphic solution with zero order of Eq. (1.11) and $\xi, o, v$ be three constants such that $\xi$, o cannot vanish simultaneously. Then

(i) $Y(z)$ has infinitely many poles.

(ii) For any finite value $B$, if $\xi \neq 0$, then $Y(z)-B$ has infinitely many zeros.

(iii) If $\xi=0$ and $Y(z)-A$ has finite zeros, then $A$ is a solution of $3 z^{2}-o-v z=0$.

We assume that the reader is familiar with the basic notions of Nevanlinna theory (see, e.g., $[5,6])$.

Theorem 1.2 Let $c \in \mathbb{C} \backslash\{0\},|\varpi| \neq 1$, and $V(z)=\frac{X(z)}{B(z)}$ be an irreducible rational function, where $X(z)$ and $B(z)$ are polynomials with $\operatorname{deg} X(z)=x$ and $\operatorname{deg} B(z)=b$.

(i) Suppose that $x \geq b$ and $x-b$ is zero or an even number. If the equation

$$
Y(\varpi z)+Y(z)+Y\left(\frac{z}{\varpi}\right)=\frac{V(z)}{Y(z)}+c
$$

has an irreducible rational solution $Y(z)=\frac{I(z)}{J(z)}$, where $i(z)$ and $J(z)$ are polynomials with $\operatorname{deg} i(z)=i$ and $\operatorname{deg} J(z)=j$, then

$$
i-j=\frac{x-b}{2}
$$

(ii) Suppose that $x<b$. If Eq. (1.13) has an irreducible rational solution $Y(z)=\frac{i(z)}{J(z)}$, then $Y(z)$ satisfies one of the following two cases: 
(1) $Y(z)=\frac{i(z)}{J(z)}=\frac{c}{3}+\frac{T(z)}{D(z)}$, where $T(z)$ and $D(z)$ are polynomials with $\operatorname{deg} T(z)=t$ and $\operatorname{deg} D(z)=d$, and $b-x=d-t$.

(2) $i-j=x-b$.

Theorem 1.3 Let $Y(z)$ be a transcendental meromorphic solution with zero order of Eq. (1.12) and $\xi, o, v$ be three constants such that $\xi$, o cannot vanish simultaneously. Then

(i) $Y(z)$ has infinitely many poles.

(ii) For any finite value $B$, if $\xi \neq 0$ and $v \neq 0$, then $Y(z)-B$ has infinitely many zeros.

(iii) If $\xi=0$ and $Y(z)-A$ has finite zeros, then $A$ is a solution of $2 z^{2}-o z-v=0$.

Theorem 1.4 Let $\xi, o, \pi$ be constants with $\xi \pi \neq 0$ and $|\varpi| \neq 1$. Suppose that a rational function

$$
Y(z)=\frac{F(z)}{U(z)}=\frac{\mu_{0} z^{m}+\mu_{1} z^{m-1}+\cdots+\mu_{m}}{\lambda_{0} z^{n}+\lambda_{1} z^{n-1}+\cdots+\lambda_{n}}
$$

is a solution of (1.12), where $F(z)$ and $U(z)$ are relatively prime polynomials, $\mu_{0} \neq 0$, $\mu_{1}, \ldots, \mu_{m}$, and $\lambda_{0} \neq 0, \lambda_{1}, \ldots, \lambda_{n}$ are constants. Then $n=m+1$ and $\mu_{0}=-\frac{\pi}{\xi} \lambda_{0}$.

\section{Some lemmas}

Lemma 2.1 ([1]) Let $Y(z)$ be a non-constant zero order meromorphic solution of

$$
Y(z)^{n} P(z, Y)=Q(z, Y)
$$

where $P(z, Y)$ and $Q(z, Y)$ are $\varpi$-difference polynomials in $Y(z)$. If the degree of $Q(z, Y)$ as a polynomial in $Y(z)$ and its $\varpi$-shifts is at most $n$, then

$$
m(r, P(z, Y))=o(T(r, Y))
$$

on a set of logarithmic density 1.

Lemma 2.2 ([1]) Let $Y(z)$ be a non-constant zero order meromorphic solution of

$$
H(z, Y)=0
$$

where $H(z, O)$ is a $\varpi$-difference polynomial in $Y(z)$. If $H(z, Y) \not \equiv 0$ for a slowly moving target $a(z)$, then

$$
m\left(r, \frac{1}{Y-a}\right)=o(T(r, Y))
$$

on a set of logarithmic density 1.

Lemma 2.3 ([7]) Let $Y(z)$ be a zero order meromorphic function, and $\varpi \in \mathbb{C} \backslash\{0\}$. Then

$$
\begin{aligned}
& T(r, Y(\varpi z))=(1+o(1)) T(r, Y(z)) \\
& N(r, Y(\varpi z))=(1+o(1)) N(r, Y(z)) .
\end{aligned}
$$




\section{Proof of Theorem 1.1}

(i): Suppose that $Y(z)$ is a zero order transcendental meromorphic solution of (1.11). By (1.11), we have

$$
Y(z) P(z, Y)=Q(z, Y)
$$

where $P(z, Y)=Y(\varpi z)+Y(z)+Y\left(\frac{z}{\varpi}\right), Q(z, Y)=\xi z+o+v Y(z)$. Lemma 2.1 implies that

$$
m(r, P(z, Y))=o(T(r, Y))
$$

on a set of logarithmic density 1. By the Valiron-Mohon'ko theorem, we obtain that

$$
T\left(r, Y(\varpi z)+Y(z)+Y\left(\frac{z}{\varpi}\right)\right)=T(r, Y)+S(r, Y)
$$

By Lemma 2.3, we obtain

$$
\begin{aligned}
N\left(r, Y(\varpi z)+Y(z)+Y\left(\frac{z}{\varpi}\right)\right) & \leq N(r, Y(\varpi z))+N(r, Y)+N\left(r, Y\left(\frac{z}{\varpi}\right)\right) \\
& =3(1+o(1)) N(r, Y) .
\end{aligned}
$$

(3.2), (3.3), and (3.4) imply that

$$
T(r, Y) \leq 3(1+o(1)) N(r, Y)+S(r, Y)
$$

on a set of logarithmic density 1 . Hence, $Y(z)$ has infinitely many poles.

(ii): For any finite value $B$, and let

$$
Y_{1}(z)=Y(z)-B
$$

Substituting $Y_{1}(z)=Y(z)-B$ into (3.1), we obtain

$$
\left(Y_{1}(z)+B\right)\left(Y_{1}(\varpi z)+Y_{1}(z)+Y_{1}\left(\frac{z}{\varpi}\right)+3 B\right)=\xi z+o+v\left(Y_{1}(z)+B\right) .
$$

Let

$$
P\left(z, Y_{1}(z)\right)=\left(Y_{1}(z)+B\right)\left(Y_{1}(\varpi z)+Y_{1}(z)+Y_{1}\left(\frac{z}{\varpi}\right)+3 B\right)-\xi z-o-v\left(Y_{1}(z)+B\right) .
$$

If $\xi \neq 0$, by (3.6), we have $P(z, 0)=3 B^{2}-\xi z-o-v B \not \equiv 0$. Lemma 2.2 implies that

$$
m\left(r, \frac{1}{Y_{1}}\right)=o\left(T\left(r, Y_{1}\right)\right)
$$

on a set of logarithmic density 1 . Hence

$$
N\left(r, \frac{1}{Y-B}\right)=N\left(r, \frac{1}{Y_{1}}\right)=T\left(r, Y_{1}\right)(1+o(1))=T(r, Y)(1+o(1))
$$

on a set of logarithmic density 1 . Hence, $Y(z)$ has infinitely many finite values. 
(iii): If $\xi=0$ and $B$ is not a solution of $3 z^{2}-o-v z=0$, then $P(z, 0)=3 B^{2}-o-v B \not \equiv 0$. Using a similar method as above, we can obtain that

$$
N\left(r, \frac{1}{Y-B}\right)=T(r, Y)(1+o(1))
$$

which contradicts the assumption of Theorem 1.1, hence the conclusion holds.

\section{Proof of Theorem 1.2}

By $(1.13)$ and $Y(z)=\frac{I(z)}{J(z)}$, we have

$$
\begin{aligned}
& B(z) I(z) I(\varpi z) J(z) J\left(\frac{z}{\varpi}\right)+B(z) I^{2}(z) J\left(\frac{z}{\varpi}\right) J(\varpi z)+B(z) I(z) I\left(\frac{z}{\varpi}\right) J(\varpi z) J(z) \\
& -c B(z) I(z) J(\varpi z) J\left(\frac{z}{\varpi}\right) J(z)=X(z) J(\varpi z) J\left(\frac{z}{\varpi}\right) J^{2}(z) .
\end{aligned}
$$

Obviously, we have

$$
\begin{aligned}
& \operatorname{deg}\left(B(z) I(z) I(\varpi z) J(z) J\left(\frac{z}{\varpi}\right)+B(z) I^{2}(z) J\left(\frac{z}{\varpi}\right) J(\varpi z)\right. \\
& \left.+B(z) I(z) I\left(\frac{z}{\varpi}\right) J(\varpi z) J(z)\right)=b+2 i+2 j \\
& \operatorname{deg}\left(c B(z) I(z) J(\varpi z) J\left(\frac{z}{\varpi}\right) J(z)\right)=b+i+3 j \\
& \operatorname{deg}\left(X(z) J(\varpi z) J\left(\frac{z}{\varpi}\right) J^{2}(z)\right)=x+4 j .
\end{aligned}
$$

(i): Suppose first that $x>b$ and $x-b$ is an even number. If $\operatorname{deg} i(z)=i<j=\operatorname{deg} J(z)$, then (4.1)-(4.4) imply that $x+4 j=b+i+3 j$, that is, $0>i-j=x-b>0$. This is impossible.

If $i=j$, then we use a similar method as above, we can obtain $0<x-b=i-j=0$, this is impossible. So, $i>j$. By (4.1), we have $x+4 j=b+2 i+2 j$, that is, $i-j=\frac{x-b}{2}$.

(ii): Suppose that $x<b$. If $i>j$, then (4.1)-(4.4) yield that $x+4 j=b+2 i+2 j$, that is, $0>x-b=2(i-j)>0$, which is a contradiction.

If $i=j$, then we can assume

$$
Y(z)=\iota_{0}+\frac{T(z)}{D(z)}
$$

where $\iota_{0} \neq 0, T(z)$ and $D(z)$ are polynomials, and $\operatorname{deg} T(z)=t<\operatorname{deg} D(z)=d$. Thus, as $z \rightarrow$ $\infty,(1.13)$ and (4.5) imply that

$$
3 \iota_{0}(1+o(1))=\frac{o(1)}{\iota_{0}(1+o(1))}+c
$$

which implies $\iota_{0}=\frac{c}{3}$. Hence,

$$
Y(z)=\frac{c}{3}+\frac{T(z)}{D(z)} .
$$


Substituting (4.7) into (1.13), we get

$$
\begin{aligned}
& B(z)\left(\frac{c}{3} D(z)+T(z)\right)\left(T(z) D(\varpi z) D\left(\frac{z}{\varpi}\right)+T(\varpi z) D(z) D\left(\frac{z}{\varpi}\right)\right. \\
& \left.+T\left(\frac{z}{\varpi}\right) D(z) D(\varpi z)\right)=X(z) D^{2}(z) D(\varpi z) D\left(\frac{z}{\varpi}\right) .
\end{aligned}
$$

Obviously,

$$
\begin{aligned}
& \operatorname{deg}\left[B ( z ) ( \frac { c } { 3 } D ( z ) + T ( z ) ) \left(T(z) D(\varpi z) D\left(\frac{z}{\varpi}\right)+T(\varpi z) D(z) D\left(\frac{z}{\varpi}\right)\right.\right. \\
& \left.\left.\quad+T\left(\frac{z}{\varpi}\right) D(z) D(\varpi z)\right)\right]=3 d+b+t \\
& \operatorname{deg} X(z) D^{2}(z) D(\varpi z) D\left(\frac{z}{\varpi}\right)=x+4 d .
\end{aligned}
$$

Hence, $b-x=d-t$.

If $i<j$, by $i<j, x<b,(4.1)-(4.4)$, then we have

$$
i-j=x-b .
$$

\section{Proof of Theorem 1.3}

(i). Suppose that $Y(z)$ is a zero order transcendental meromorphic solution of (1.12). By (1.12), we have

$$
Y^{2}(z)\left(Y(\varpi z)+Y\left(\frac{z}{\varpi}\right)\right)=(\xi z+o) Y+v
$$

Lemma 2.1 implies that

$$
m\left(r, Y(\varpi z)+Y\left(\frac{z}{\varpi}\right)\right)=o(T(r, Y))
$$

on a set of logarithmic density 1 . By the Valiron-Mohon'ko theorem, we get that

$$
T\left(r, Y(\varpi z)+Y\left(\frac{z}{\varpi}\right)\right)=T(r, Y)+S(r, Y)
$$

By Lemma 2.3, we obtain

$$
N\left(r, Y(\varpi z)+Y\left(\frac{z}{\varpi}\right)\right) \leq N(r, Y(\varpi z))+N\left(r, Y\left(\frac{z}{\varpi}\right)\right)=2(1+o(1)) N(r, Y)
$$

(5.2), (5.3), and (5.4) yield that

$$
T(r, Y) \leq 2(1+o(1)) N(r, Y)+S(r, Y)
$$

on a set of logarithmic density 1 . Hence, $Y(z)$ has infinitely many poles. 
(ii). For any finite value $B$, let

$$
Y_{1}(z)=Y(z)-B
$$

Substituting $Y_{1}(z)=Y(z)-B$ into (5.1), we obtain

$$
\left(Y_{1}(z)+B\right)^{2}\left(Y_{1}(\varpi z)+Y_{1}\left(\frac{z}{\varpi}\right)+2 B\right)=(\xi z+o)\left(Y_{1}(z)+B\right)+v
$$

Let

$$
P\left(z, Y_{1}(z)\right)=\left(Y_{1}(z)+B\right)^{2}\left(Y(\varpi z)+Y\left(\frac{z}{\varpi}\right)+2 B\right)-(\xi z+o)\left(Y_{1}(z)+B\right)-v .
$$

By (5.6), we have $P(z, 0)=2 B^{2}-(\xi z+o) B-\pi$.

If $B=0$ and $\pi \neq 0$, then we obtain that $P(z, 0)=-v \not \equiv 0$.

If $B \neq 0$, then we have $P(z, 0)=2 B^{2}-(\xi z+o) B-v \not \equiv 0$ since $\xi \neq 0$. Using a method similar to Theorem 1.1, we can obtain that

$$
N\left(r, \frac{1}{Y-A}\right)=N\left(r, \frac{1}{Y_{1}}\right)=T\left(r, Y_{1}\right)(1+o(1))=T(r, Y)(1+o(1))
$$

on a set of logarithmic density 1 . Hence, $Y(z)$ has infinitely many finite values.

(iii). If $\xi=0$ and $A$ is not a solution of $2 z^{2}-o z-\pi=0$, then using a method similar to Theorem 1.1, we also obtain that

$$
N\left(r, \frac{1}{Y-A}\right)=T(r, Y)(1+o(1))
$$

which contradicts the assumption of Theorem 1.3, hence the conclusion holds.

\section{Proof of Theorem 1.4}

Assume that (1.12) has a rational solution $Y(z)$ and has poles $t_{1}, t_{2}, \ldots, t_{k}$. Next, let

$$
\frac{c_{j \lambda_{j}}}{\left(z-t_{j}\right)^{\lambda_{j}}}+\cdots+\frac{c_{j_{1}}}{\left(z-t_{j}\right)} \quad(j=1, \ldots, k)
$$

be the principal parts of $Y$ at $t_{j}$, respectively, where $c_{j \lambda_{j}}, \ldots, c_{j 1}$ are constants, $c_{j \lambda_{j}} \neq 0$. Hence

$$
Y(z)=\frac{F(z)}{U(z)}=\sum_{j=1}^{k}\left[\frac{c_{j \lambda_{j}}}{\left(z-t_{j}\right)^{\lambda_{j}}}+\cdots+\frac{c_{j_{1}}}{\left(z-t_{j}\right)}\right]+\tau_{0}+\tau_{1} z+\cdots+\tau_{s} z^{s}
$$

where $\tau_{0}, \ldots, \tau_{s}$ are constants. Assume that $\tau_{s} \neq 0(s \geq 1)$. When $z \rightarrow \infty$,

$$
\begin{aligned}
& Y(z)=\tau_{s} z^{s}(1+o(1)), \quad Y(\varpi z)=\varpi^{s} \tau_{s} z^{s}(1+o(1)), \\
& Y\left(\frac{z}{\varpi}\right)=\frac{1}{\varpi^{s}} \tau_{s} z^{s}(1+o(1)) .
\end{aligned}
$$


By (1.12), we have

$$
Y^{2}(z)\left(Y(\varpi z)+Y\left(\frac{z}{\varpi}\right)\right)=(\xi z+o) Y+v .
$$

When $z \rightarrow \infty$, (6.2), (6.3), and (6.4) imply that

$$
\left(\varpi^{s}+\frac{1}{\varpi^{s}}\right) \tau_{s}^{3} z^{3 s}(1+o(1))=(\xi z+o)\left(\tau_{s} z^{s}(1+o(1))\right)+v
$$

which is a contradiction since $\tau_{s} \neq 0$ and $s \geq 1$. Assume that $\tau_{0} \neq 0$, as $z \rightarrow \infty$,

$$
\begin{aligned}
& Y(z)=\tau_{0}(1+o(1)), \quad Y(\varpi z)=\tau_{0}(1+o(1)), \\
& Y\left(\frac{z}{\varpi}\right)=\tau_{0}(1+o(1)) .
\end{aligned}
$$

By (6.4) together with (6.5) and (6.6), we obtain that

$$
(\xi z+o)\left(\tau_{0}(1+o(1))\right)=2 \tau_{s}^{3}-\pi
$$

This is impossible since $\xi \neq 0$ and $\tau_{0} \neq 0$. Hence

$$
Y(z)=\frac{F(z)}{U(z)}
$$

where $\operatorname{deg} F(z)=m<\operatorname{deg} U(z)=n$. Equation (6.7) and (1.12) imply that

$$
\begin{aligned}
& F^{2}(z) F(\varpi z) U\left(\frac{z}{\varpi}\right)+F^{2}(z) F\left(\frac{z}{\varpi}\right) U(\varpi z) \\
& \quad=(\xi z+o) F(z) U(z) U(\varpi z) U\left(\frac{z}{\varpi}\right)+v U^{2}(z) U(\varpi z) U\left(\frac{z}{\varpi}\right) .
\end{aligned}
$$

Hence we have $n=m+1$. By (1.12) and $n=m+1$, we obtain

$$
\frac{F(\varpi z)}{U(\varpi z)}+\frac{F\left(\frac{z}{\varpi}\right)}{U\left(\frac{z}{\varpi}\right)}=\frac{(\xi z+o) F(z) U(z)+v U^{2}(z)}{F^{2}(z)}
$$

Since as $z \rightarrow \infty$, we have

$$
\frac{F(\varpi z)}{U(\varpi z)}+\frac{F\left(\frac{z}{\varpi}\right)}{U\left(\frac{z}{\varpi}\right)} \rightarrow 0
$$

and

$$
\frac{(\xi z+o) F(z) U(z)+v U^{2}(z)}{F^{2}(z)}=\frac{\left(\xi \mu_{0} \lambda_{0}+v \lambda_{0}^{2}\right) z^{2 n}(1+o(1))}{\mu_{0}^{2} z^{2 n-2}(1+o(1))},
$$

we obtain $\xi \mu_{0} \lambda_{0}+\pi \lambda_{0}^{2}=0$. 
Funding

The work was supported by the NNSF of China (No. 10771121, 11301220, 11401387, 11661052), the NSF of Zhejiang Province, China (No. LQ 14A010007), the NSF of Shandong Province, China (No. ZR2012AQ020), and the Fund of Doctoral Program Research of Shaoxing College of Art and Science (20135018).

\section{Competing interests}

The authors declare that there is no conflict of interest regarding the publication of this paper

Authors' contributions

All authors contributed equally and significantly in writing this paper. All authors read and approved the final manuscript.

Author details

${ }^{1}$ Department of Mathematics, Shaoxing College of Arts and Sciences, Shaoxing, China. ${ }^{2}$ Archive, Shaoxing College of Arts and Sciences, Shaoxing, China.

\section{Publisher's Note}

Springer Nature remains neutral with regard to jurisdictional claims in published maps and institutional affiliations.

Received: 1 February 2018 Accepted: 15 July 2018 Published online: 15 August 2018

\section{References}

1. Barnett, D.C., Halburd, R.G., Korhonen, R.J.: Nevanlinna theory for the q-difference operator and meromorphic solutions of q-difference equation. Proc. R. Soc. Edinb., Sect. A, Math. 137, 457-474 (2007)

2. Chen, Z.X.: On properties of meromorphic solutions for some difference equations. Kodai Math. J. 34, 244-256 (2011)

3. Chen, Z.X., Shon, K.H.: Value distribution of meromorphic solutions of certain difference Painlevé equations. J. Math. Anal. Appl. 364, 556-566 (2010)

4. Halburd, R.G., Korhonen, R.: Finite order solutions and the discrete Painlevé equations. Proc. Lond. Math. Soc. 94 443-474 (2007)

5. Hayman, W.K.: Meromorphic Functions. Clarendon Press, Oxford (1964)

6. Yang, C.C., Yi, H.X.: Uniqueness Theory of Meromorphic Functions. Kluwer Academic, Dordrecht (2003)

7. Zhang, L.J., Korhonen, R.: On the Nevanlinna characteristic of $f(q z)$ and its applications. J. Math. Anal. Appl. 369, 537-544 (2010)

\section{Submit your manuscript to a SpringerOpen ${ }^{0}$ journal and benefit from:}

- Convenient online submission

Rigorous peer review

Open access: articles freely available online

High visibility within the field

Retaining the copyright to your article 